\title{
An Identification of Faults in Industries Using Hybrid Feed Forward Neural Network - Particle Swarm Optimization
}

Anoopa Jose Chittilappilly ( $\square$ anoopajosephd1@gmail.com )

Karpagam University

Kamalraj Subramaniam

Karpagam University

Giby Jose

Karpagam University

P. Manimegalai

Karpagam University

\section{Research Article}

Keywords: non-local means, feed forward neural network classifier, particle swarm optimization.

Posted Date: April 21st, 2021

DOl: https://doi.org/10.21203/rs.3.rs-391761/v1

License: (1) This work is licensed under a Creative Commons Attribution 4.0 International License.

Read Full License 


\section{An Identification of Faults in Industries using Hybrid Feed Forward Neural}

\section{Network - Particle Swarm Optimization}

Anoopa Jose Chittilappilly ${ }^{1}$, Dr. Kamalraj Subramaniam ${ }^{2}$

Giby Jose $^{3}$, and Dr. P. Manimegalai ${ }^{4}$

${ }^{1}$ Department Electronics \& Communication Engineering, Karpagam University,

Coimbatore, Tamil Nadu, India. Email: anoopajosec@yahoo.co.in

${ }^{2}$ Department Electronics \& Communication Engineering, Karpagam University,

Coimbatore, Tamil Nadu, India. Email:kamalrajece@gmail.com

${ }^{3}$ Department Electronics \& Communication Engineering, Karpagam University,

Coimbatore, Tamil Nadu, India. Email: gibyjose77@yahoo.com

${ }^{4}$ Department Electronics \& Communication Engineering, Karpagam

University,Coimbatore, Tamil Nadu, India.Email: manimegalai.vairavan@gmail.com

\section{Abstract:}

In order to enhance the quality governance in automotive firms the fault analysis and categorization is designed with optimal image renewals employing swarm intelligence scheme with improved precision classifier. Methodology: Preliminarily the accumulated information is preprocessed for eradicating the undesirable noise and renewal is achieved employing non - local means scheme, followed by which five characteristics like arithmetic mean, variance, standard deviation, skewness and auto correlation are mined. The mined characteristics are sent to the feed forward neural network (FFNN) classifier for recognizing faults in the computerized segments produced in the firms. In FFNN the particle swarm optimization (PSO) is employed to optimize the characteristics for effective fault identification in metal sheets. Results: The experimental analysis reveals that the designed FFNN - PSO scheme acquires 
improved performance with increased rate of accuracy of $92.86 \%$, sensitivity rate of $95.24 \%$, specificity rate of $90.48 \%, \mathrm{G}-$ mean rate of $97.47 \%$ and precision rate of $90.90 \%$ evaluated against the prevailing classifiers.

Keywords:non-local means, feed forward neural network classifier, particle swarm optimization.

\section{Introduction}

Generally, quality control is a crucial issue in metal sheet manufacturing method. So, the assembly examination ought to perform to judge the merchandise. Today, in most of sheet product lines, quality control is performed manually by knowledgeable folks. The shortage of an automatic quality control system causes reduction in potency, lack of adequate preciseness, and increasingexpenditure. Image processing is that dominant technology within the field for inspecting completely different textures and recognizing accessible diversity. Capability of this technology, particularly in these two contexts of detection and categorizing the sample, opens the chance to utilize this approach for internal quality control in industries like textile, paper, ceramic etc.Now a good deal of analysis has been created in automatic detection of defects on the surface

of metal sheets. ${ }^{1}$ Surface degradation is that issue that causes almost $70 \%$ of machine failure. Therefore, it's vital to understand and classify the defects in surfaces mechanically at early stages of production. Customers deliberately seek for objects within the surroundings with a linear texture and observe the course of reflection lines on the surface of the body. Studies ${ }^{2}$ showed that dents and bumps with already 10 micrometers depth and a number of other millimeters lateral dimension are often visually perceived on a painted car-body. Within the early production stages, these little 
defects are hidden from the human observer, due to the rough, boring surface quality of the raw components. They become visible during the stages when the components get painted which means that they become mirrorlike reflective. The later the defect gets detected, a lot of correction can price. Currently, the raw sheet components are solely indiscriminately tested and mistreated in typical ways e.g. using gauge, inspection byspecialists, abrasive uncovering of the components and observation of the scratch marks, or highlight the surface with special oil that enables perception of the reflection lines as a result of oil film's specularity. These ways are time overwhelming and supported by human observations, entailing perspicacity, lack of repeatability and quantitativity.

Coordinate measurement machines (CMM) will, though, turn out correct, quantitative results, however as a result of the mechanical contact with the versatile half, the results are biased and also the measurement method will take more than 10 minutes just in case of few points and not to mention the necessity for special environmental conditions. Laser-triangulation techniques have a bonus in responding speed over CMMs, though measure of a surface still desires many scanning steps. Fringe projection methodology will at the same time capture several surface points of sunshine coloured, homogeneous, texture-less, diffuse reflective objects, but glossy, metallic surfaces have to be compelled to be treated by a special chalk-spray previously to cut back the gloss, otherwise it generates wheezy or no measure information in any respect. Deflectometry could be a white lightweight, whole field methodology, which is suitable for the shape measure of reflective; extremely reflective objects e.g. mirrors, lenses, or painted car-body components, while raw, uninteresting surfaces need to be treated with oil so as to 
get radiancy. The necessity for surface treatment like highlighting using oilor applying chalk spray hinders within the production, and when final product is obtained, the components need to be clean leading to removal of the homogeneously treated one result in necessity for excessive use of such substances which makes it neither economical nor ecological.

A new, whole-field measure methodology is required, which may sight and numerically measure little deviations of the surface. The first detection of surface defects on raw sheet components could be a crucial downside within the automotive business. Typical defects like dents, bumps, and waviness are invisible at the first production stages due to the parts' rough surfaces. The defects show and heavy solely once the later production steps during which the components get painted and painted, which means that they become specular-reflecting. The later the defect gets detected, a lot of correction can price. Currently, the raw sheet components are solely indiscriminately tested by specially trained specialists, which mean the results are subjective and qualitative. To overcome this problem, we propose novel classifier using FFNN-PSO classifier which reduce the detection time and cost. Initially, the five feature of the input image is obtained then it will be trained by the proposed FFNN-PSO classifier where it detects the defect in the metal sheet.

\section{Related Work}

Most of the defect detection systems are disbursed in Gray-level images. Thresholding or edge detection techniques are utilized to detect defects in non-textured surfaces like glass panels, sheet steel etc.For sophisticated textured surfaces in gray-level images, gray-level co-occurrence matrix ways ${ }^{3,4}$ within the spatial domain and Fourier remodel ways within the frequency domain are usually wont to describe 
textural options. Within the previous years, some defect detection techniques are projected. The data of those detection techniques has been collected from varied resource materials. ${ }^{5}$ M. Ghazvini, A. Monadjemi, K. Jamshidi uses detail matrices that contains median, max and min points for defect detection. ${ }^{6}$ J.L .Sobral stressed on ripple sub band and optimized Gabor filters for texture defect detection. ${ }^{7}$ Hamid Alimohamadi highlighted the employment of filter banks and optimized filter for defect detection and extraction of feature image. ${ }^{8}$ Ajay Kumar and Grantham Pang discovered Gabor filters may be deployed for material defect detection along with Bernoulli's rule of combination to integrate information. ${ }^{9}$ Kaicheng yin and Weidong Yu used segmentation for defect detection in clothes production system. ${ }^{10}$ Henry Y. T .Ngan, Grantham K. H. Pang, S. P. Yung, Michael K. nanogram projected patterned cloth defect detection using golden image subtraction methodology. ${ }^{11}$ Jun Xie, YifengJiang,Hung-tat Tsui projected the employment of segmentation technique in medical application. ${ }^{12}$ Yi-leng Chen, Tse-Wei Chen,Shao-Yi Chien discovered however wavelet transform may be used for quick texture feature extraction. ${ }^{13}$ J. P. Yun, S. H. Choi, S. W. Kim projected an algorithm using Laplacian and edge conserving filter for real time defect detection for Bar in Coil. ${ }^{14}$ J. W. Kim , S. W. Kim developed a method which uses Gabor Filteroptimized by univariate dynamic encoding algorithm for searches (uDEAS). The algorithm finds the minimum price associated with the energy separation criteria between the defect and also the defect-free regions. ${ }^{15}$ C. H. Park, S. H. Choi, S. W. Won highlighted the employment of wavelet remodel for periodic defects in steel wire rod production. ${ }^{16}$ D. C. Choi, Y. J. Jeon, J. P. Yun projected an algorithm using Gabor Filter and morphological features for detecting pinholes in steel 
slabs. ${ }^{17}$ Paulraj M P, KamalrajSubramaniam, Sazali Bin Yaccob, Abdul Hamid Bin Adom, Hema C R suggested that from the neural network models, it has been inferred that they were able to classify the normal hearing and abnormal hearing subjects with conductive or sensorineural hearing loss. ${ }^{18}$ Anoopa Jose Chittilappilly and KamalrajSubramaniamproposed Waveletneural networks for industrial applications in automotive defect detection system.

\section{Materials and Methods}

Fig. 1shows the algorithm for defect detection and classification. The target of defect detection is raw metal sheet. The image is acquired using a line scan camera. The flow starts with assortment of images of metal plates acquired from trade. Non Local means method is used for restoration. Thenfeatures are extracted.Classifier is used to judge whether or not defect is present.In this section, we propose a defect detection and classification technique for metal plates so that we can achieve higher defect detection rate with reduced computational time. Sequences of operation for proposed algorithm are as follows. 


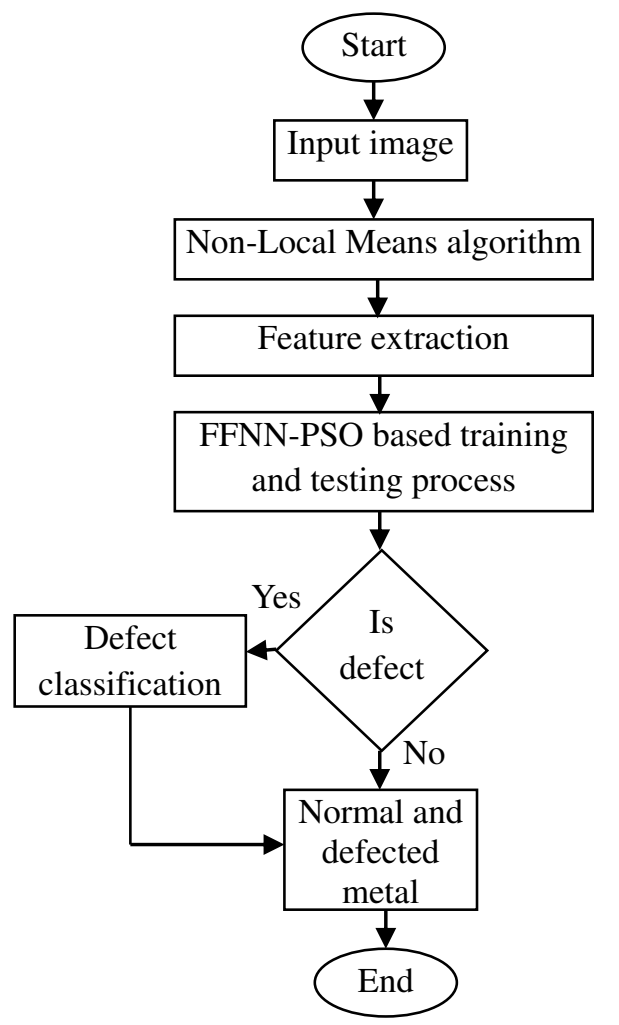

Fig.1: overall process of proposed scheme

\subsection{Feature extraction}

In this section five features such as Mean, Variance, Standard deviation, Skewness and Autocorrelation are extracted and the description is given as follows.

\subsubsection{Arithmetic mean}

The mean filter, additionally called averaging filter, operates on an sliding ' $\mathrm{m} \times \mathrm{n}$ ' window by scheming the common of all component values within the window and exchanging the middle component value within the destination image with the result. Its mathematical formulation is given as follows as

$$
f(i, j)=\frac{1}{m n} \sum_{(r, c) \varepsilon w} g(r, c)
$$

\subsubsection{Variance}

The variance is a measure of how far a set of numbers is spread out. It is one of several descriptors of a probability distribution, describing how far the numbers lie from the 
mean (expected value). In particular, the variance is one of the moments of a distribution. In that context, it forms part of a systematic approach to distinguish between probability distributions. While other such approaches have been developed, those based on moments are advantageous in terms of mathematical and computational simplicity. Mathematically variance is given by

$$
f(i, j)=\frac{1}{m n-1} \sum_{(r, c) \varepsilon w}\left(g(r, c)-\frac{1}{m n} \sum_{(r, c) \varepsilon w} g(r, c)\right)^{2}
$$

\subsubsection{Standard deviation}

It is a most generally used measure of variability or diversity utilized in statistics. In terms of image processing it shows what proportion variation or "dispersion" exists from the common (mean, or expected value). An occasional standard deviation indicates that the information points tend to be very close to the mean, whereas high standard deviation indicates that the information points are detached over an oversized variety of values. Mathematically standard deviation is given by

$$
f(i, j)=\sqrt{\frac{1}{m n-1}} \sum_{(r, c) \varepsilon w}\left(g(r, c)-\frac{1}{m n} \underset{(r, c) \varepsilon w}{\left.\sum_{r} g(r, c)\right)^{2}}\right.
$$

\subsubsection{Skewness}

In statistics, skewnesscould be a measure of the imbalance of the likelihood distribution of a real-valued variable. The asymmetry price may be positive or negative, or perhaps undefined. Mathematically asymmetry may be given by

$$
\mathrm{f}(\mathrm{i}, \mathrm{j})=\frac{\frac{1}{\mathrm{mn}-1} \Sigma(\mathrm{r}, \mathrm{c}) \in \mathrm{W}\left(\frac{1}{\mathrm{mn}-1} \Sigma(\mathrm{r}, \mathrm{c}) \in \mathrm{W} \mathrm{g}(\mathrm{r}, \mathrm{c})-\frac{1}{\mathrm{mn}} \Sigma(\mathrm{r}, \mathrm{c}) \in \mathrm{W}(\mathrm{r}, \mathrm{c})\right)^{3}}{\left(\frac{1}{\mathrm{mn}-1} \Sigma(\mathrm{r}, \mathrm{c}) \in \mathrm{W}\left(\frac{1}{\mathrm{mn}-1} \Sigma(\mathrm{r}, \mathrm{c}) \in \mathrm{W} \mathrm{g}(\mathrm{r}, \mathrm{c})-\frac{1}{\mathrm{mn}} \Sigma(\mathrm{r}, \mathrm{c}) \in \mathrm{W} \mathrm{g}(\mathrm{r}, \mathrm{c})\right)^{2}\right)^{3 / 2}}
$$




\subsubsection{Autocorrelation}

Autocorrelation itself logically resembles comparing all potential component pairs and news the probability that each is going to be bright as a function of the distance and direction of separation. Duringan additional mathematical definition, autocorrelation is that the convolution of a function with itself. For a digital image I, of size $\mathrm{M} \times \mathrm{N}$ (images are discretely spatially outlined, 2- to four-dimensional, of finite extent, and have real, bounded, digital values), autocorrelation may be calculated by equation given below.

$G_{x x}(a, b)=\sum_{i}^{M} \sum_{j}^{N} x(i, j) * x(i-a, j-b)$

(5)where $G_{x x}(a, b)$ is the autocorrelation function, $x(i, j)$ is the image intensity at position (i,j), and a and $\mathrm{b}$ represent the distance (or lag) from the corresponding $i$ and $j$ position.

\subsection{FFNN-PSO Based Defect Detection and Classification}

Once the network topology is specified, corresponding weights of every connecting node have to be compelled to be trained. Within the study, the full weights of the network are going to be thought of because the position vector of the particle withinthe Particle Swarm optimization (PSO) and therefore the weights of every particle vector are initialized randomly and are evolved by the PSO rule.During this study, PSO is applied to train feed forward neural network for enhancing the convergence rate and learning method. The essential component of $\mathrm{NN}$ is neuron. Every neuron is joined with its neighbors with an associated weight that represents data employed to resolve a problem. The training method involves finding a collection of weights that minimizes the training error. The position of every particle in FFNN-PSO represents a collection of 
weight for current iteration ${ }^{19}$. The dimension of every particle is the range of weights related to the network. The error of this network is computed by Mean square Error (MSE). The training method of FFNN-PSO is given below.

Step 1:The training method of FFNN-PSO is initialized with a gaggle of random particles, which were appointed with random PSO positions (weight and bias).

Step 2:The FFNN-PSO is trained for the initial particles position.

Step 3:Then, the feed forward NN in FFNN-PSO can turn out the training error (particle fitness) supported initial weight and bias. The training error at current epoch is going to be reduced by dynamic particle position, which is able to update the load and bias of the network.

Step 4:The "pbest" value (each particle's lowest learning error) and "gbest" value (lowest learning error found in entire learning method) are applied to the velocity update equationfor position adjustment to the simplest solution or targeted learning error.

Step 5:The new sets of positions (NN weight and bias) are made by adding the calculated velocity value to the present position value equation. Then, the new sets of positions are wont to turn out new learning error in feed forward NN.

Step 6:This method is recurrent till the stopping conditions either minimum learning error or most range of iteration are met. The improved output was supported gbest position value. The particle position represents two-dimensional (2D) vector of $\mathrm{x}$ and $\mathrm{y}$ values in Sombrero operate. The target is to succeed in the valueof one supported value of $\mathrm{x}$ and $\mathrm{y}$ in Sombrero equation (Eq. 6) and therefore the goal for the PSO is to maximize the operate. 
$z=6 * \cos \left(\frac{\sqrt{x * x+y * y}}{x * x+y * y+6}\right)$

where $\mathrm{x}$ is value in $\mathrm{x}$-axis, $\mathrm{y}$ is value in $\mathrm{y}$-axis, $\mathrm{z}$ is value in $\mathrm{z}$-axis. The fitness of the particle on the Sombrero is represented by z-axis. The overall classification is shown in Fig 2.

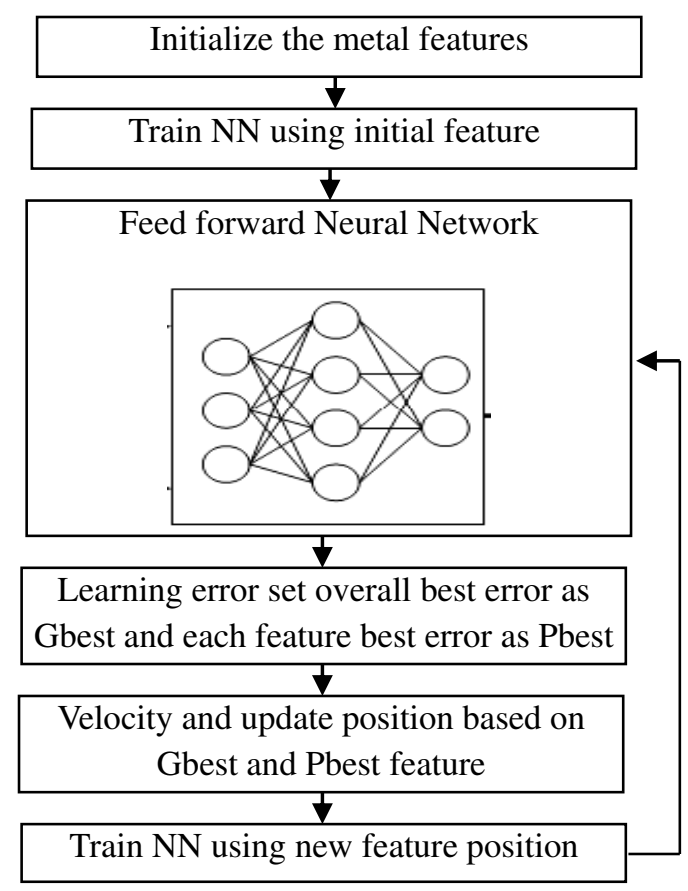

Fig.2: overall process of classification

\section{Results and Discussion}

In this section, the overall performance of proposed FFNN-PSO has been evaluated in terms of sensitivity, specificity, accuracy, G-mean and precision. The image restoration has been performed using Non Local Means algorithm. Fig. 3 shows the simulated result of a defectivematerial. The results are shown in Table 1.

With the increase in number of data, the accuracy value increases linearly. The accuracy value of the proposed FFNN-PSO algorithm is observed to be higher. This is mainly due to the optimum feature selection. The sensitivity and specificity of proposed scheme is also high. The graphical representation of this algorithm is given in Fig. 4. 


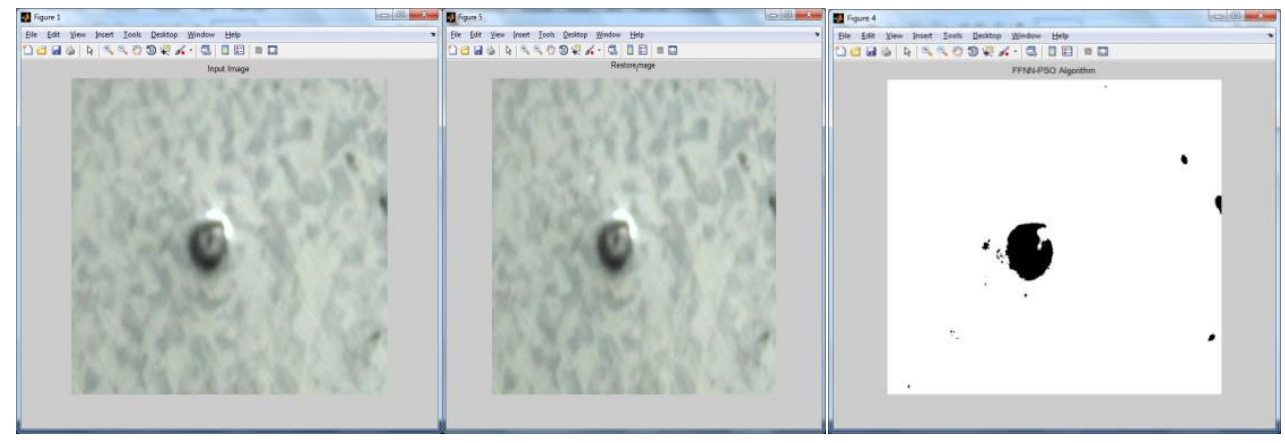

(a)

(b)

(c)

Fig.3: (a) Input Image; (b) Restored Image(c) Segmented Images

Table 1: Results of FFNN-PSO Classification

\begin{tabular}{|c|c|c|c|c|c|}
\hline Class & Accuracy & Sensitivity & Specificity & Precision & G-mean \\
\hline Normal sheet 1 & 92.03 & 95.43 & 89.96 & 88.78 & 96.67 \\
\hline Normal sheet 2 & 92.12 & 94.78 & 90.32 & 89.98 & 97.65 \\
\hline Defective sheet 1 & 92.34 & 95.43 & 89.98 & 90.21 & 96.34 \\
\hline Defective sheet 2 & 92.43 & 95.34 & 90.23 & 90.78 & 95.54 \\
\hline Defective sheet 3 & 92.87 & 95.21 & 90.34 & 90.87 & 97.65 \\
\hline Average & 92.8571 & 95.2381 & 90.4762 & 90.9091 & 97.4679 \\
\hline
\end{tabular}

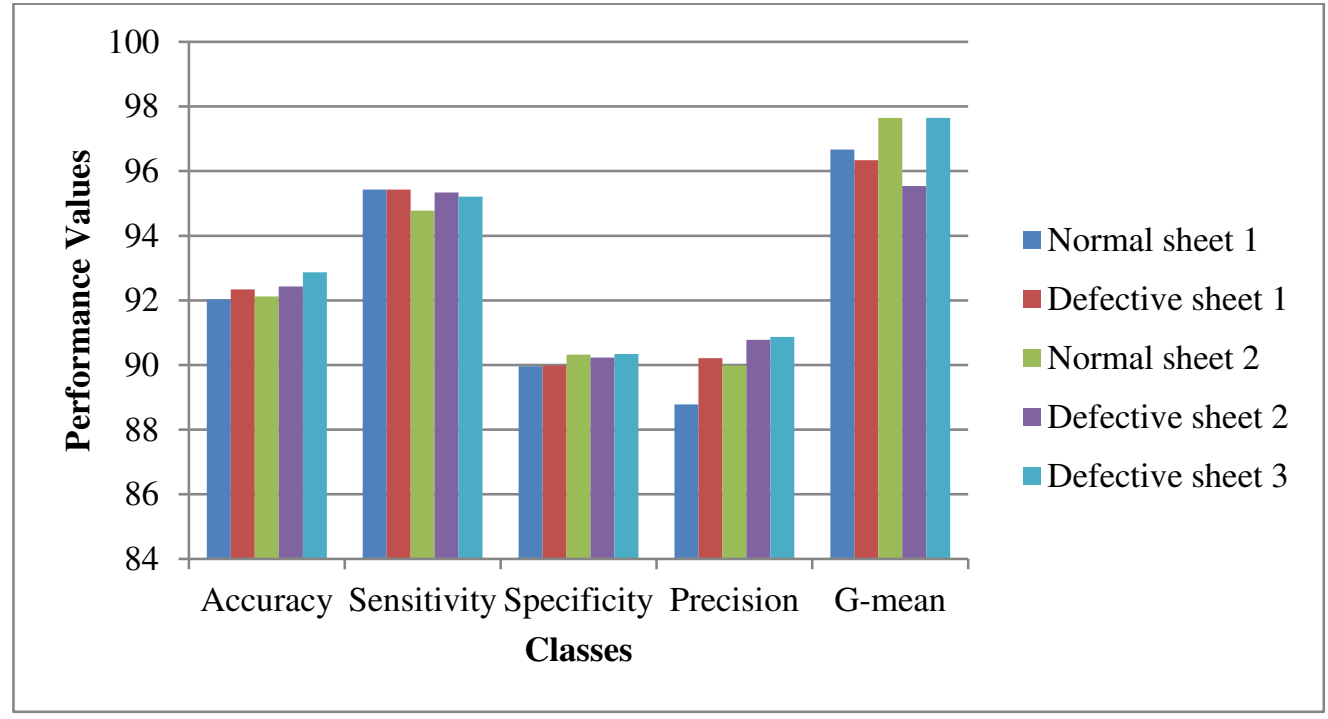

Fig.4: performance evaluation for various classes 


\section{Conclusion}

Here the identification of faults such as spots and scrapes on the metal sheets are based on the FFNN - PSO scheme. The scheme could be employed for diverse sorts of images and the designed scheme is helpful in escalating the speed of identification along with the management of precision while identification. The scheme could be employed for assessing other sorts of products like plastic, wood and fabrics. It is anticipated that the system aids the steel firms to enhance the reliability of the product quality and minimizing the expenses in manufacturing.

\section{Conflict of interest:}

There is no conflict of interest.

\section{Funding information:}

There is no funding information.

\section{Availlability of data and material:}

There is no availability of data and material.

\section{Code availability:}

There is no code availability.

\section{Author's contribution:}

Theree is no author's contribution.

\section{References}

[1] He XD, Torrance KE, Sillion FX, Greenberg DP. A comprehensive physical model for light reflection. ACM SIGGRAPH Computer Graphics. 1991; 25(4):175-186.

[2] Kühn B, Hrabal V, Brückner S, Lang GK, Traue HC. Physiologie und Psychologie der Wahrnehmung von Geometriefehlern auf Karosserieoberflächen. Universität Ulm; 1994. 
[3] MostafaSadeghi, FaezehMemarzadehzavareh, "Flaws detection in steel plates Using Gabor Wavelet”, Life Science Journal, pp.352-355, 2013.

[4] T. Aarthi, M. Karthi, M. Abinesh "Detection and analysis of surface defects in metals using wavelet transform", International Journal of Scientific and Research Publications, Volume 3, Issue 6, June 2013.

[5] M. Ghazini, A. Monadjemi and K. Jamshidi, "Defect detection of tiles using 2D Wavelet transform and statistical features", World Academy of Science, Engineering \& Technology, Vol. 49, pp. 901-904, 2009.

[6] J.L. Sobral, “Optimized filters for texture defect detection" IEEE International conference on Image Processing, pp.565-573, 2005.

[7] Hamid Alimohamdi and AlirezaAhmady, "Detecting skin defect of fruits using optimal Gabor wavelet filter", International conference on Digital image Processing, pp.402-406, 2009.

[8] Ajay Kumar and Grantham Pang, "Defect Detection in textural materials using Gabor filters" IEEE Transactions on Industry Applications, Vol. 38, No. 2, pp. 425-440, 2002.

[9] Yin, Kaicheng Yu and Weidong, "Image processing for the use of garment production detection System”, Congress on Image and Signal Processing, Vol. 3, pp. 349-352, 2008.

[10]Henry Y.T. Ngan, Grantham K.H. Pang, S.P. Yung and Michael K. Ng, "Wavelet based methods on patterned fabric defect detection", Pattern Recognition, Vol. 38, No. 4, pp. 559-576, 2005. 
[11]Jun Xie, Yifeneg Jiang and Hung-tat Tsai, "Segmentation of kidney from ultra sound images based on texture and shape priors", IEEE Transactions of Medical Imaging, Vol.24, No.1, pp. 45-57, 2005.

[12] Yiling Chen, TseWei Chen and Shaoyichen, "Fast texture feature extraction method based on segmentation for image retrieval", 13th IEEE International Symposium on Consumer Electronics, pp. 941-942, 2009

[13]J. P. Yun, S. H. Choi, S. W. Kim," Real time vision based defect inspection for high speed steel products”,OptEng.47(5),2008 pp.1-8.

[14]J. P. Yun, , J. W. Kim, S. W. Kim “Automatic detection of cracks in raw steel block using Gabor filter using univariate dynamic encoding algorithm for searches (uDEAS)", NDT\&E International, 42, 2009, pp.389-397.

[15]C. H. Park, S. H. Choi, S. W. Won "Vision based inspection for periodic defects in steel wire rod production", Opt. Eng 49(1), 2010, 017202, pp. 1-10.

[16]D. C. Choi, Y. J. Jeon, J. P. Yun Pinhole detection in steel slab images using Gabor filter and morphological features", applied optics, 51(26), 2011, pp. 5122-5129.

[17]Paulraj M P, KamalrajSubramaniam, Sazali Bin Yaccob, Abdul Hamid Bin Adom, Hema C R, "EEG Based Detection of Conductive and Sensorineural Hearing loss using Artificial Neural Networks," Journal of Next generation Information Technology, Vol 4, Issue 3, pp. 204-212, 2013, South Korea.

[18]Anoopa Jose Chittilappilly and KamalrajSubramaniam, "WNN in Image Restoration for Industrial Applications", International Journal of Computer Applications 154(10):17-23, November 2016. 
[19]Chun-Feng Wang and Kui Liu, A Novel Particle Swarm Optimization Algorithm for Global Optimization, Computational Intelligence and Neuroscience Volume 2016 (2016), Article ID 9482073, 9 pages. 
Figures

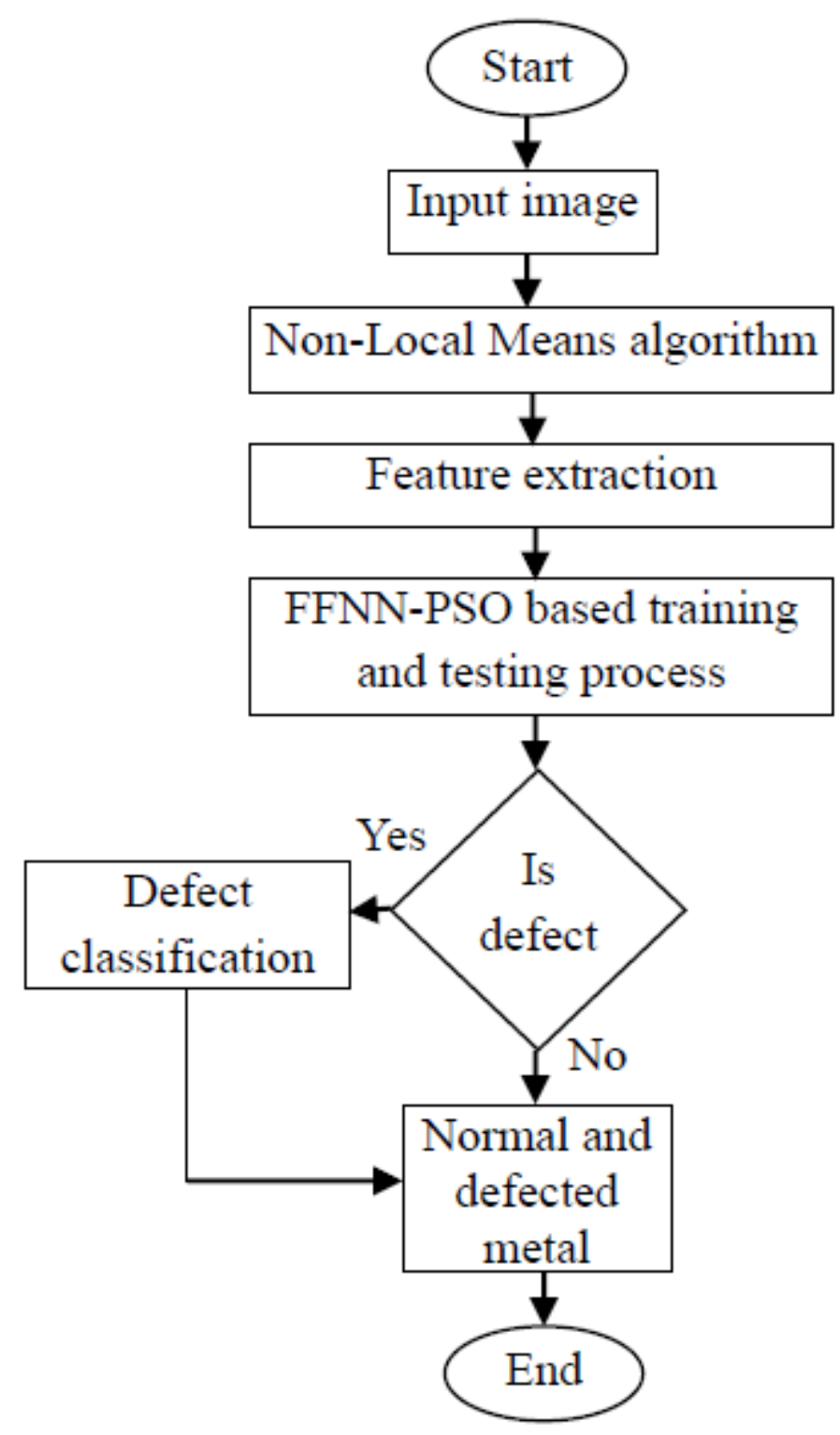

Figure 1

overall process of proposed scheme 


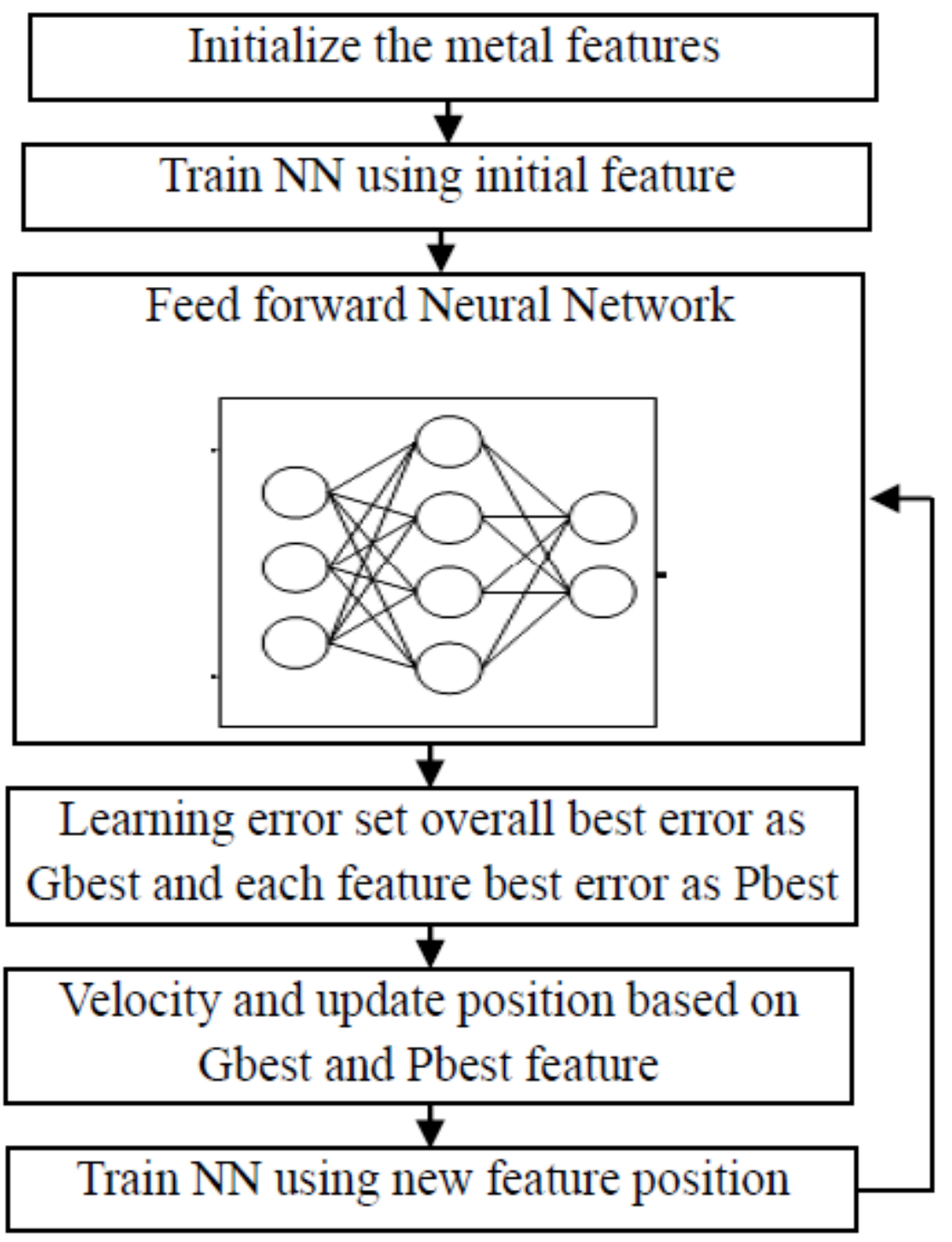

Figure 2

overall process of classification 


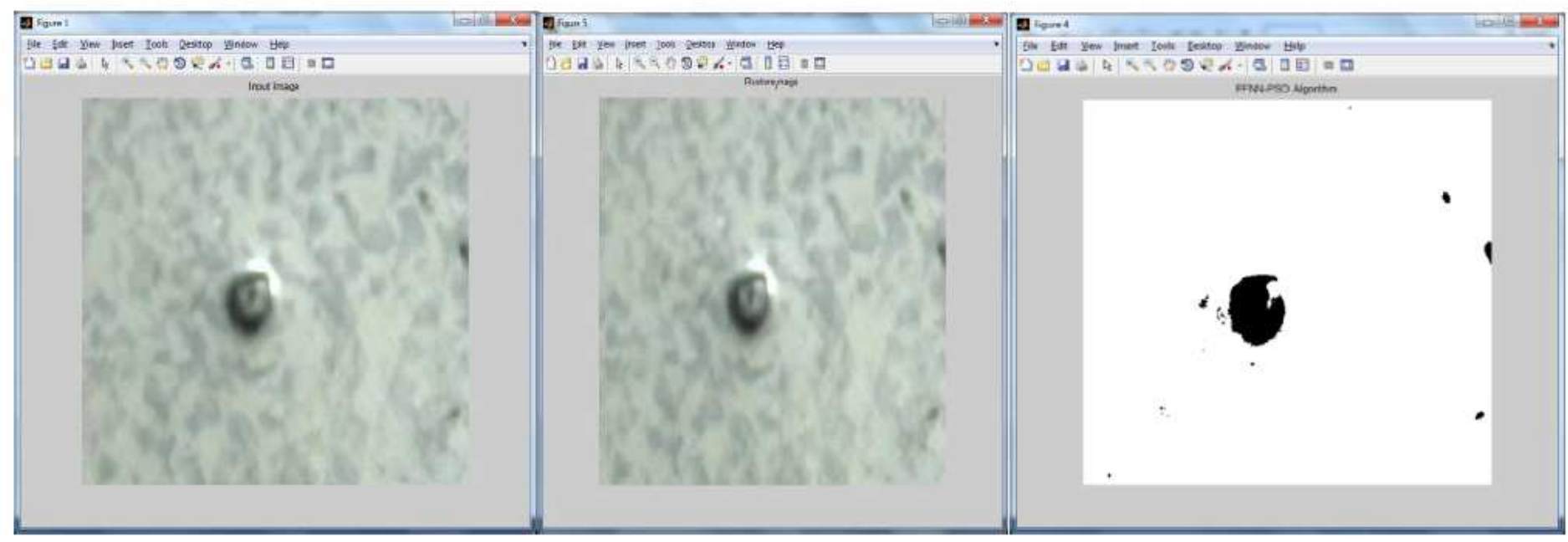

(a)

(b)

(c)

Figure 3

(a) Input Image; (b) Restored Image; (c) Segmented Images

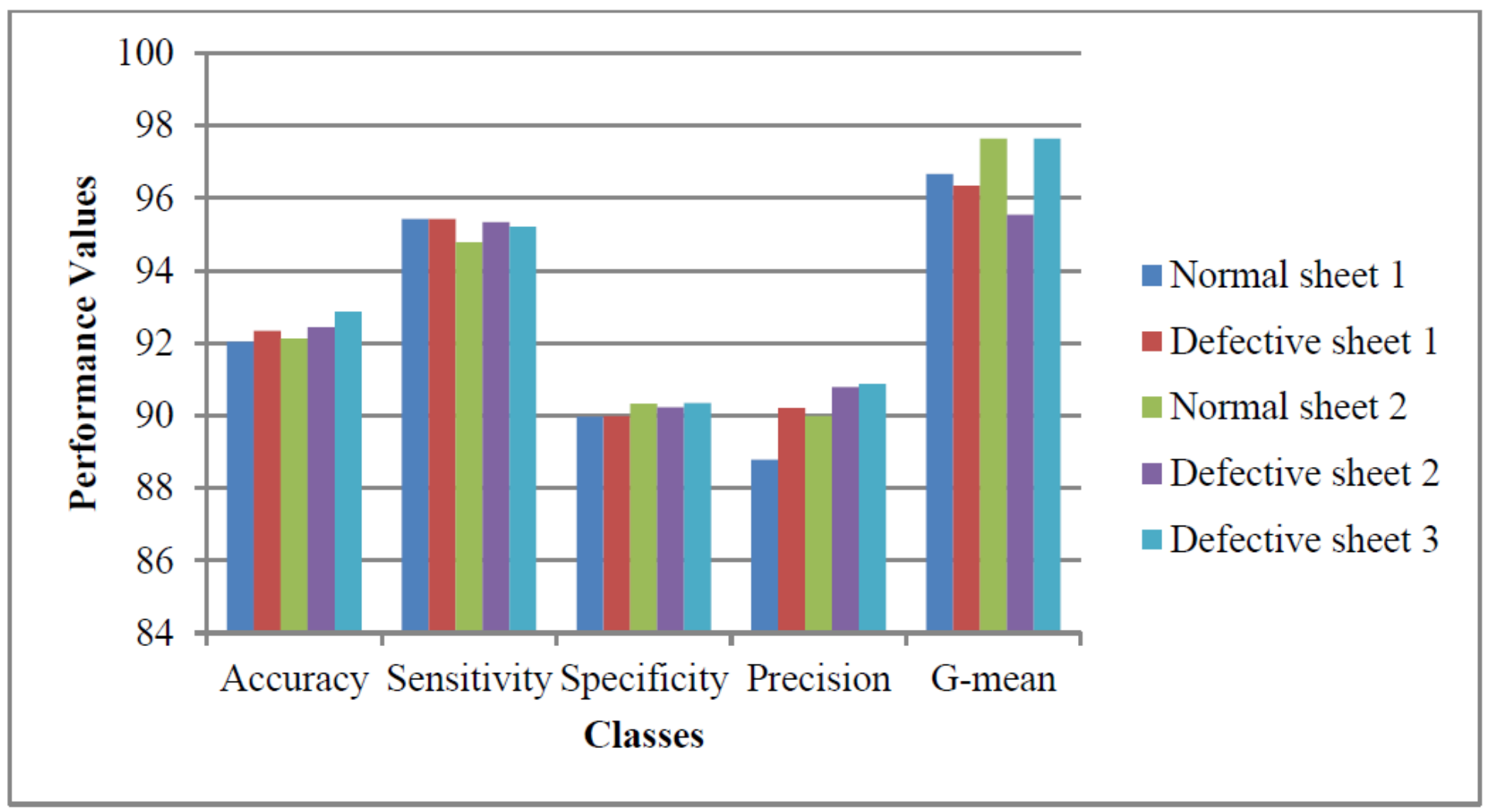

Figure 4

performance evaluation for various classes 